then the Levy-Broughton-Taylor mention of the simple and excellent example of 1000-100-100-100 vs. 999-998997-996 still seems to be telling.

When asked about implementation, I had proposed one, although acknowledging that the case might be so rare that it would not justify the extra evaluation time. But I heartily agree with the authors that the lack of such an algorithm is a striking difference between computers' and human play.

Your publication of this letter will correct the historical perspective of the origin of the suggestion."

THE 50-MOVE RULE ADAPTED (1)

\author{
Edmar Mednis
}

$41-4273$ rd Street

Woodside, New York 11377 / USA

\begin{abstract}
"As a follow-up to my article in issue \#1, 1989 (p. 30-36), I would like to inform the readers that FIDE at its November 1988 meeting lowered the " 50 move" rule requirements from the previous 100 moves to 75 moves. In my opinion this is still too long."
\end{abstract}

\title{
THE 50-MOVE RULE ADAPTED (2)
}

\author{
B.M. Kažić \\ President of FIDE Rules Commission \\ Generala Zdanova 21 \\ Belgrade, Yugoslavia
}

"The Rules Commission of FIDE has taken into consideration the results of computer analyses of some endgames which were published by computer-chess experts; first of all we looked at the results published in the ICCA Journal. I have distributed some copies of your Journal among the members of our commission.

The Grand Master Association (GMA) also recommended to accept a new version of the 50-move rule. However, the GMA had the idea of deciding by cases, i.e., allowing for distinct endings a different number of moves. The Commission's opinion was that it would be better and simpler for arbiters to accept one maximal number of 75 moves in exceptional cases."

[In the next issue, the Journal will return to the topic of the 50-move rule. - Eds.] 